\title{
POLÍTICAS PÚBLICAS EN CIENCIA Y TECNOLOGÍA EN MÉXICO. GOBIERNO 2018-2024.
}

\author{
PUBLIC POLICIES IN SCIENCE AND TECHNOLOGY IN MEXICO. GOVERNMENT \\ 2018-2024.
}

\author{
Mijael Altamirano-Santiago ${ }^{1}$ (iD . \\ 1. Centro de Investigaciones Económicas Administrativas y Sociales \\ Instituto Politécnico Nacional. \\ *Correspondencia del Autor: Mijael Altamirano-Santiago, correo electrónico: maltamiranos@ipn.mx.
}

\section{RESUMEN}

Objetivo: El objetivo principal es dar cuenta del cauce de la política púbica en Ciencia y Tecnología (C\&T) en México, con especial referencia, en el gobierno 2018-2024. Material y Método: Teoría de la representación social (Moscovici, 1979) a efecto de explicar a detalle lo que hoy día se publica, se trasmite o se divulga sobre la C\&T. Discusión: Se expone el cauce que ha tenido la C\&T en México, teniendo presente sus componentes, tales como: gastos, fondos institucionales, estímulos fiscales, capital humano, programa institucional. Conclusión: Se reporta que la C\&T no se ha tenido un trato especial en los asuntos públicos, tampoco se ha configurado una política pública de Estado en C\&T.

Palabras clave : Ciencia; tecnología; políticas públicas; teoría de la representación social.

Cómo citar:

Altamirano-Santiago, Mijael. (2021). Políticas públicas en Ciencia y Tecnología en México. Gobierno 2018-2024. Revista de Investigaciones Universidad del Quindio, 33(1), 06-23. https:// doi.org/10.33975/riuq.vol33n1.441

Información del artículo: Recibido: 11 febrero 2021; Aceptado: 19 febrero 2021 


\section{ABSTRACT}

Objective: The main objective is to account for the course of public policy in Science and Technology (S\&T) in Mexico, with special reference to the 2018-2024 government. Material and Method: Theory of social representation (Moscovici, 1979) in order to explain in detail what is published, transmitted or disseminated about S\&T today. Discussion: The course of S\&T in Mexico is presented, bearing in mind its components, such as expenditure, institutional funds, fiscal incentives, human capital, institutional program. Conclusion: It is reported that S\&T have not been given special treatment in public affairs, neither has a State public policy on science and technology been configured.

Keywords: Science; technology; public policies; theory of social representation.

\section{INTRODUCCIÓN}

Hace ya más de cinco décadas en que la UNESCO recomendó a los países en vías de desarrollo invertir el 1\% de su PIB en Ciencia y Tecnología (C\&T), toda vez que un impulso a este binomio permitiría a las naciones encauzar su dinámica con miras a mantener un crecimiento sostenido y emerger con ventajas favorables de cara al proceso global.

En efecto, es así, si se tiene presente el caso de Corea del Sur (también conocido como República de Corea) que en esa misma década en que la UNESCO hizo tal recomendación, inició por ejecutar políticas públicas encaminadas a fortalecer su mercado y su estructura productiva, sin olvidar que este binomio C\&T debía configurarse como una estrategia de Estado, hasta alcanzar lo que actualmente es este país en el concierto de las naciones. Según SaKong (2010, p. 22) "en los últimos 60 años la República de Corea no solo ha logrado un espectacular crecimiento económico, sino también un desarrollo social de una amplia base", incluso en palabras de Altamirano (2010) este país es capaz de financiar proyectos científicos y tecnológicos más allá de sus fronteras.

Por ello no es casual, que las sociedades postindustriales (Toffler, 1980; Ritzer, 2007) de cara a la globalización le den especial trato al binomio C\&T (y ahora sumada la innovación) para constituirse en el trinomio $\mathrm{C} \& \mathrm{~T}+\mathrm{I}$ a efecto de posicionarse como economías competitivas y con sobradas ventajas frente a sus similares.

Este esquema que han adoptado algunos países, no esta lejos de lo que en su momento sugirió Solow (1956), quien desarrolló la teoría del crecimiento económico identificando, entre su planteamiento, un factor exógeno (el progreso tecnológico) como un impulsor del crecimiento de la economía, incluso - señalan otros autores como Barro y Sala-i-Martin (1995), Blanchard y Fischer (1989) o Romer (1996) — este mismo componente exógeno se configura como un elemento para lograr ventajas competitivas y una creciente acumulación de capacidades.

La realidad que experimentan aquellos países que basan su prosperidad económica en las ventajas que trae invertir en $\mathrm{C} \& \mathrm{~T}+\mathrm{I}$ o, en su caso, asumir políticas públicas fundadas en la economía del conocimiento (Sakaya, 1985; Stigitz, 1999; Bassand, 2001; Grant, 1996; Powell \& Snellman, 2004) son hoy en día naciones desarrolladas y con resultados altamente alentadores que los ha llevado a posicionarse frente a sus pares con un estatus preponderante.

Justamente, es así, la institucionalización de una política pública encaminada a consolidar la C\&T + I o una economía basada en el conocimiento ha marcado el curso de acción de las naciones en el proceso global, incluso ha conducido que 
existan distancias entre los países desarrollados y los no desarrollados.

México, si bien es cierto que en su momento tuvo una economía creciente entre los años 30 y 60 , no es así en los tres últimos sexenios, en donde el crecimiento ha sido de magro (en promedio $2 \%$ ) y, más aún, en los últimos 2 años (2019-2020) en donde más que estancamiento, ha sido una recesión económica (en promedio -10.5), teniendo su punto más bajo en el segundo trimestre de 2020 (-18.7\%), lo que debió haber obligado a los gobiernos en turno, inclusive el actual, mirar a otros horizontes a efecto de conocer las experiencias de otras latitudes o, al menos, atender lo dicho por la UNESCO, para darle al país un viraje en sus indicadores nacionales.

De ahí, que el presente artículo tiene como objetivo principal dar cuenta del cauce de la política púbica en C\&T en México, con especial referencia, en el gobierno 2018-2024.

\section{METODOLOGÍA}

El tratamiento de una realidad presente (sea social, económica o política) o, en su caso, dar cuenta del acontecer de los actores e instituciones en un régimen político, debe necesariamente tener como base un método, una teoría o una técnica, a efecto de que el objeto-sujeto de investigación sea expuesto con claridad, rigor y, sobre todo, con perspicuidad de explicar el estado de la cuestión $\mathrm{y}$, de ese modo, conocer las implicaciones, alcances, cauces, entre otros, del objeto-sujeto de investigación.

De ahí, que el investigador para dar cuenta de la política pública en C\&T en México, considera la teoría de la representación social (Moscovici, 1979). Esto es así, a efecto de explicar a detalle lo que hoy día se publica, se trasmite o se divulga sobre la C\&T.

La teoría de la representación social (RS) se funda como: "(...) una modalidad particular del conocimiento, cuya función es la elaboración de los comportamientos y la comunicación entre individuos (...) una representación social es alternativamente el signo, el doble de un objeto valorizado socialmente. (...) es un corpus organizado de conocimientos y una de las actividades psíquicas gracias a las cuales los hombres hacen inteligibles la realidad física y social, se integran a un grupo o en una relación cotidiana de intercambios, libran los poderes de su imaginación" (Moscovici, 1979, p. 18).

En tanto, Piña y Cuevas (2004) y Cárdenas et al (2007), afirman que la RS es un conjunto de ideas, saberes y conocimientos para que cada persona comprenda, interprete y actúe en su realidad inmediata. La RS se teje con el pensamiento que la gente organiza, estructura y legitima en su vida cotidiana. El conocimiento es, ante todo, un conocimiento práctico que permite explicar una situación, un acontecimiento, un objeto o una idea y, además, permite a las personas actuar ante un problema (Moscovici, 1979).

\section{DISCUSIÓN}

\section{a. Una revisión de la realidad actual que experimenta la $\mathrm{C} \& \mathrm{~T}$}

Con el reciente gobierno de 2018-2024, México experimenta una nueva realidad - similar a lo acontecido con el gobierno 2000-2006- dado que por primera vez llega un partido distinto a los que tradicionalmente habían sido gobierno en la dinámica nacional.

Este gobierno recién inaugurado empezó su periplo desde hace un poco más de 20 años como Jefe de Gobierno en el Distrito Federal (hoy Ciudad de México) y eso daba pie a la sociedad en general para pensar que el país emergería en un nuevo cauce que introdujera nuevos referentes en el quehacer de las instituciones y del propio gobierno. De igual modo, un sector social (académicos, intelectuales, especialistas, jóvenes, entre otros) que había desconfiado, incluso había mostrado hartazgo, sobre el curso que tomaba las políticas públicas en el país, vislumbraban 
nuevas formas y fondos en el proceso de toma de decisiones y en el accionar de los poderes públicos.

Esto es así, si se tiene en cuenta la declaración de principios del partido de gobierno (MORENA, 2014) que señala a la letra: "En el México actual, la vida política e institucional está marcada por la corrupción, la simulación y el autoritarismo. A pesar de ello, millones de mexicanos trabajan a diario honesta y arduamente, practican la solidaridad y se organizan para acabar con este régimen caduco. Con esas premisas, nació en 2010 el Movimiento Regeneración Nacional (MORENA), que hoy como partido político busca la transformación democrática del país. Un cambio en lo político, económico, social y cultural. Esto se logrará imprimiendo principios éticos a nuestra organización y defendiendo los derechos humanos, la libertad, la justicia y la dignidad de todos" (p. 1).

Todo ello, inyectó nuevos bríos a la realidad nacional $\mathrm{y}$, más aún, con lo estipulado en la declaración de principios de MORENA (2014) en donde refiere que se "valora el conocimiento y el aprendizaje de las experiencias, tradiciones científicas y culturales propias y de otros pueblos, particularmente los de América Latina y el Caribe. Nuestro movimiento está comprometido con la creación y las libertades, con la educación de calidad en la libertad y para la libertad. El arte y la cultura son territorios privilegiados para renovar las esperanzas en un futuro mejor, más humano y más igualitario" (p. 5).

Así, con el partido de gobierno (MORENA) se percibían nuevos cauces en la dinámica económica, social, política, educativa, científica, tecnológica, entre otros rubros, dado que la experiencia marcada por sus antecesores (Partido Revolucionario Institucional (PRI) y Partido de Acción Nacional (PAN), poco o nada modificaron la ruta nacional, ya que éste exigía la implementación de políticas públicas (Bardach, 1999) eficientes, eficaces y efectivas para propiciar condiciones prósperas y ventajas comparativas (Porter, 1990; Ricardo, 1993; Smith, 2019; Smith, 2018) para el país.

\section{b. Gasto Federal en Ciencia y Tecnología (GFCyT)}

Los gobiernos de antaño propiciaron avances significativos en diversas áreas de gobierno, aunque no suficientes y, menos aún, en lo referente al GFCyT que por costumbre y jerarquía en la toma de decisiones siempre quedó en un plano secundario, sea por los intereses de gobierno, por la cuestión ideológica, por las prioridades políticas de la partidocracia (Sartori, 2005) o, en su caso, por la escala que tenía la C\&T en la estructura organizativa del Estado, pues a través de un análisis sincrónico (comparado) se observa que todo este tiempo los recursos asignados a este binomio ha oscilado por debajo de $0.5 \%$.

Imagen 1. Evolución del gasto en Ciencia y Tecnología del gobierno federal (como porcentaje del PIB y como porcentaje del gasto total).

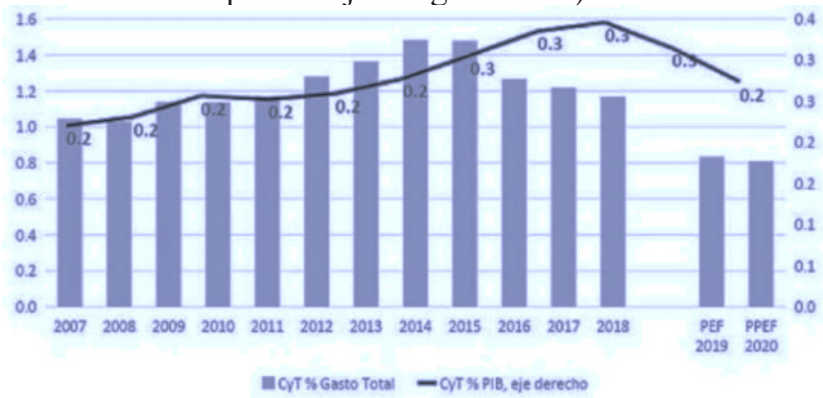

Fuente: Ruelas (2019).

1 El Gasto Federal en Ciencia y Tecnología (GFCyT) es el conjunto de erogaciones que por concepto de gasto corriente, inversión física, inversión financiera, así como pago de pasivos o deuda pública, realizan las Secretarías de estado y los departamentos administrativos; la Procuraduría General de la República; los organismos públicos autónomos; los organismos descentralizados; las empresas de control presupuestario directo e indirecto; los fideicomisos en los que los fideicomitentes sea el Gobierno Federal para el financiamiento de las actividades científicas y tecnológicas, principalmente. El gasto comprende tres actividades científicas y tecnológicas: i) investigación y desarrollo experimental (IDE), ii) educación y enseñanza científica y técnica (formación de recursos humanos a nivel de posgrado) y iii) servicios científicos y tecnológicos. 


\section{c. El horizonte de la C\&T}

El horizonte de la C\&T con la alternancia política se percibía creciente y con mayor dinamismo, realidad que no es así. En efecto, afirma Ruelas (2019) cuando refiere “(...) las cifras de gasto demuestran que sucede lo contrario a lo que genuinamente se esperaría. El desarrollo de la C\&T no ha sido, y se vislumbra que tampoco lo será, una prioridad ni del Estado y en consecuencia tampoco del sector privado. No existe un liderazgo institucional que apueste por la transformación productiva" (párrafo, 7).

La comunidad científica esperaba un viraje en el trato de este binomio porque representaba desde la visión del partido de gobierno un apartado especial al afirmar que se "valora el conocimiento y el aprendizaje de las experiencias, tradiciones científicas" (MORENA, 2014, p. 5), incluso representaba una oportunidad para México y, en consecuencia, para todos los sectores sociales, empresariales, productivas, académicas, entre otros, para apuntalar una agenda pública que generara cursos de acción en sus áreas respectivas.

De aquí que el sector académico sugirió darle más margen a la $\mathrm{C} \& \mathrm{~T}$, no sólo en el plano presupuestal, sino también promover nuevas áreas y acciones en su contexto $\mathrm{y}$, sobre todo, impulsar una política de Estado para avanzar de manera sistemática y ordenada en este ámbito. La respuesta para tal efecto no la hubo en el aspecto cuantitativo, ni cualitativo.

En lo referente a la parte cuantitativa, la asignación presupuestal en C\&T sigue siendo marginal como antaño, sin poder superar la media porcentual, inclusive en estos dos años del presente sexenio (2018-2024) guarda la misma tendencia al posicionarse alrededor de $0.2 \%$ (imagen 1). Misma situación experimenta la parte cualitativa, toda vez que la política pública diseñada y ejecutada cambió al limitar en número las áreas disciplinares del Sistema Nacional de Investigadores (SNI), dado que antes de 2020, las áreas estaban estructuradas del modo siguiente:
"Área I: físico matemáticas y ciencias de la tierra; Área II: biología y química; Área III: ciencias médicas y de la salud; Área IV: humanidades y ciencias de la conducta; Área V: ciencias sociales; Área VI: biotecnología y ciencias agropecuarias; Área VII: ingenierías; Comisión transversal de tecnología" (SNI-CONACYT, 2019). Posteriormente a este año, el Área VI. Biotecnología y Ciencias Agropecuarias, ya no aparece como tal. Desapareció como área. Situación que fue criticada por la comunidad científica, académicos y diversas Organizaciones de la Sociedad Civil.

Según Roldán (2020) en su columna afirma "Desde el lunes pasado la comunidad científica expresó su preocupación por este cambio en el reglamento. Este jueves la Sociedad Mexicana de Biotecnología y Bioingeniería (SMBB) emitió una carta en la que rechazó que se eliminara esta área del conocimiento y pidió que se incluyera de nuevo en el documento. La Mesa Directiva Nacional de la SMBB externa su preocupación y rechaza la eliminación de la biotecnología como área del conocimiento en el nuevo Reglamento del Sistema Nacional de Investigadores publicado en el Diario Oficial de la Federación el 21 de septiembre de 2020 (...) En otra carta, 42 investigadores de diversas universidades del país como la UNAM, la UAM y el Cinvestav demandaron que se restituya a la Biotecnología dentro de las áreas del conocimiento del SNI. Destacaron que actualmente existen más de 9 mil profesionales activos en biotecnología en México, quienes representan una tercera parte de la investigación nacional. Asimismo, que en el país se ofrecen 614 programas de estudio a nivel licenciatura que tienen un enfoque en la biotecnología, así como 320 posgrados con una matrícula superior a los 7 mil inscritos".

El propio Consejo Nacional de Ciencia y Tecnología (CONACYT, 2020) afirmó sobre este tema al señalar que "La disciplina de biotecnología no desapareció en el nuevo Reglamento del Sistema Nacional de Investigadores, publicado en el DOF el pasado 21 de septiembre de 2020. 
Al contrario: ante la diversidad de líneas de investigación de la comunidad de biotecnología, se permite ingresar su solicitud en tres diferentes áreas del SNI, dependiendo de la pertinencia: Área II. Biología y Química; Área VII. Ciencias de Agricultura, Agropecuarias, Forestales y de Ecosistemas; Área VIII. Ingenierías y Desarrollo Tecnológico [y] Sólo se eliminó la nomenclatura anterior. Cuestión que, de manera desinformada, ha querido ser utilizada para confundir a la opinión pública".

Más allá de este ajuste, lo real es que en vez de desagregar aún más las áreas a efecto de considerar la especificidad de la producción científica y tecnológica de la comunidad académica en un espectro más amplio y específico, se reduce o se acota sin darle el estatus que merece cada una, como tradicionalmente ha sido en el contexto global.

\section{i. Ramo 38 para el CONACYT y estímulos fiscales}

Mención aparte merece lo realizado en materia de estímulo fiscal en el sexenio 2000-2006, periodo en que se introdujo por primera vez un ajuste a la legislación en materia de C\&T:

i) Creación del Ramo 38 para el CONACYT. En este sexenio 2018-2024, de acuerdo a lo publicado por la SHCP en el Presupuesto de Egreso de la Federación (SHCP, 2021) "el Ramo 38 Consejo Nacional de Ciencia y Tecnología se alinea a la Directriz 3: Desarrollo económico incluyente, [delineado en el Plan Nacional del Desarrollo 2019-2024]. Conforme a la Misión del Ramo 38 Consejo Nacional de Ciencia y Tecnología (Ramo 38), que enuncia: Contribuir al impulso y fortalecimiento de la investigación científica, el desarrollo tecnológico y la innovación en el país, mediante la generación y aplicación de las humanidades, ciencias y tecnologías que generen una ciencia comprometida con la sociedad y el medio ambiente, y logre la soberanía científica que eleven el bienestar de la población" (p. 1).

Imagen 2. Participación relativa de los ramos en el Presupuesto del Programa de C\&T, 2013-2020.

$\begin{array}{llllllllll}\text { R11 } & \text { Educación Pública } & 21.8 \% & 21.0 \% & 21.9 \% & 26.3 \% & 29.2 \% & 34.4 \% & 39.5 \% & 42.5 \% \\ \text { R38 } & \text { CONACYT } & 34.6 \% & 39.1 \% & 40.5 \% & 43.9 \% & 33.3 \% & 33.8 \% & 31.0 \% & 29.7 \% \\ \text { R12 } & \text { Salud } & 7.5 \% & 6.8 \% & 6.8 \% & 7.5 \% & 7.0 \% & 7.3 \% & 6.9 \% & 7.4 \% \\ \text { R18 } & \text { Energía } & 8.2 \% & 8.8 \% & 8.9 \% & 9.4 \% & 8.3 \% & 7.6 \% & 7.2 \% & 6.8 \% \\ \text { R08 } & \begin{array}{l}\text { Agricultura } \\ \text { Provisiones Salariales y }\end{array} & 4.3 \% & 7.2 \% & 7.6 \% & 10.6 \% & 9.5 \% & 9.7 \% & 7.9 \% & 5.8 \% \\ \text { R23 Económicas } & 5.5 \% & 5.2 \% & 5.4 \% & 3.6 \% & 2.7 \% & 3.2 \% & 3.7 \% & 2.7 \%\end{array}$

Fuente: https://www.lja.mx/2019/11/se-reduce-0-5-el-presupuesto-al-ramo-38- para-conacyt/

Como se observa en la imagen 2, el Ramo 38 Consejo Nacional de Ciencia y Tecnología (CONACYT), la tendencia que guarda no alcanza desde 2013, ni antes de este año, el 50\% del GTCyT, por el contrario, ha seguido una línea que ronda entre el 29.7 y $43.9 \%$, siendo su caída más notoria en 2020 durante el periodo 20132020. Según nota LJA-MX (2019) “[el] Ramo 38 no sufre ningún incremento, prácticamente permanece igual en cuanto a recursos fiscales.
Sin embargo, de acuerdo con un análisis del Foro Consultivo Científico y Tecnológico, si tomamos en cuenta los recursos propios que generan las instituciones del ramo 38, éste se reduce 0.5 por ciento. El Programa Nacional de Ciencia, Tecnología e Innovación (PCTI) 2020 forma parte de los programas presupuestarios transversales del Gobierno Federal y suma 98,724.4 millones. Esta cantidad se distribuye en 18 dependencias federales, entre las que se encuentra el Conacyt, 
en lo que se conoce como Ramo 38, y el cual recibe el 29.7 por ciento del total: 29,203.2 millones de pesos; mientras que en 2019 se le asignaron 29,358.5, es decir la diferencia con respecto al año anterior es de 155.3 millones".

ii) Modificación al artículo 219 de la Ley Impuesto Sobre la Renta (30 por ciento de estímulo fiscal a empresas con inversión en Investigación y Desarrollo Experimental-IDE). Dicho artículo refiere "Se otorga un estímulo fiscal a los contribuyentes del impuesto sobre la renta por los proyectos en investigación y desarrollo tecnológico que realicen en el ejercicio, consistente en aplicar un crédito fiscal equivalente al $30 \%$ de los gastos e inversiones realizados en el ejercicio en investigación o desarrollo de tecnología, contra el impuesto sobre la renta causado en el ejercicio en que se determine dicho crédito. Cuando dicho crédito sea mayor al impuesto sobre la renta causado en el ejercicio en el que se aplique el estímulo, los contribuyentes podrán aplicar la diferencia que resulte contra el impuesto causado en los diez ejercicios siguientes hasta agotarla" (Cámara de Diputados, 2011).

Esta modificación trajo consigo a la creación del Programa de Estímulos Fiscales al Gasto en Investigación y Desarrollo de Tecnología de las Empresas Privadas en México (EFIDT) ${ }^{2}$ (PIF) al Programa de Incentivos Fiscales (PIF), actualmente denominado "Estímulo Fiscal a la Investigación y Desarrollo de Tecnología (EFIDT)" que se configura como "un estímulo otorgado a través de un crédito fiscal al contribuyente que realice gastos e inversiones en IDT, es acreditable contra el ISR de los contribuyentes y puede ser ejercido en un periodo de 10 años, hasta agotarlo. El estímulo es equivalente al 30\% de los gastos e inversiones incrementales en IDT, respecto al monto de gastos e inversiones promedio en los tres ejercicios 2 El Estímulo Fiscal consiste en un crédito fiscal de 30\% aplicable al Impuesto sobre la Renta o Impuesto al Activo, dirigido a todos los contribuyentes, personas morales o físicas con actividad empresarial que inviertan en el desarrollo de nuevos productos, procesos y servicios. anteriores al ejercicio en el cual se solicite el estímulo o un monto máximo de 50 millones de pesos". Y cuyo objetivo es "Incrementar la inversión privada en la investigación científica y desarrollo tecnológico en el País, propiciando así diversos efectos derrama positivos asociados a dicha actividad" (CONACYT, 2020a).

Y ¿cómo se acceder a este estímulo? Así lo expone El Financiero (2017, sección economía) retomando lo afirmado por Carreón "Las empresas deberán registrarse e ingresar la información fiscal de los últimos tres años de la compañía. Se requerirá declaración fiscal de 2014, 2015 y 2016 y deberán indicar cuanto invirtió en ese periodo en investigación y desarrollo".

Y ¿cómo se calcula? Continúa Calderón "Supongamos que una empresa invirtió cinco millones en 2014, 10 en 2015 y 15 en 2016, la suma de esos tres nos da 30 millones, que 1o dividimos entre tres para sacar el promedio. De tal suerte que esos 10 millones, que es el promedio de inversión de los últimos tres años, es lo que se tomará como base, y si esta empresa manifiesta que en este año invertirá 40 millones, a este monto se le restará el promedio, es decir, a los 40 millones se le quitarán 10, quedarán 30 millones, así que el estímulo fiscal será 30 por ciento de esos 30 millones (...) Es decir, la empresa tendría un crédito fiscal de aproximadamente nueve millones de pesos para pagar su impuesto sobre la renta (ISR) en 2018 cuando haga su declaración de 2017” (El Financiero, 2017, sección economía).

Tabla 1. Recursos asignados al Estímulo Fiscal a la Investigación y Desarrollo de Tecnología (EFIDT).

\begin{tabular}{c} 
2020: $\$ 105,247,216.16$ \\
2019: $\$ 406,276,997.49$ \\
2018: $\$ 331,032,847.28$ \\
2017: $\$ 658,353,350.11$ \\
\hline $\begin{array}{c}\text { Fuente: Elaboración propia con base de https://www. } \\
\text { conacyt.gob.mx/index.php/fondos-y-apoyos/estimulo- } \\
\text { fiscal-a-la-investigacion-y-desarrollo-de-Tecnología-efidt } \\
\text { (ver ejercicio fiscales) }\end{array}$ \\
\hline
\end{tabular}


Similar que el Ramo 38, los recursos asignados al EFIDT se reducen en $74.1 \%$ entre 2019 y 2020 (tabla 1). Esta realidad desalienta el quehacer de un gran número de empresas o unidades productivas en el rubro de investigación y desarrollo de tecnología (IDT), incluso se vuelve lastimoso si se tiene en cuenta la vivencia que experimentan las sectores productivos para el acceso a este EFIDT, pues además del exceso y la complejidad de los trámites administrativos, resulta poco atractivo dado que éste se otorga bajo reglas de operación que lo frena mucho más al segmentar las deducciones en IDT, programas de capacitación, desarrollo de nuevos productos, procesos y servicios; aún así el sector productivo ha incrementado un poco más sus recursos destinados a este rubro, sin llegar a ser altamente significativo comparado a sus similares en otras latitudes -Francia y España, que otorgan apoyos que ronda sobre el $0.42 \mathrm{y}$ 0.34 de descuento de impuesto por cada dólar gastado en $\mathrm{I}+\mathrm{D}-$, que además de dedicarle más recursos, las modalidades son distintas $\mathrm{y}$, sobre todo, atractivas. A esto, se suma lo afirmado por Martuscelli (2012) con base al estudio "Programa de Estímulos Fiscales" realizado por Asociación Mexicana de Directivos de la Investigación Aplicada y el Desarrollo Tecnológico (ADIAT), en donde recomienda "Modificar el sistema de evaluación actual [del EFIDT] por uno que se dedique a revisar únicamente la elegibilidad de las inversiones y su pertinencia" (p. 47).

De ahí, que la cuestión administrativa representa una limitante para los sectores productivos ya que complica en mucho el acceso a este EFIDT, por un lado, y, por el otro, el techo económico asignado a este programa. Ejemplo de este último, se observa en la imagen 3, elaborada por Dutrénit et al (2010) en donde se observa que, en el 2006, del total de las empresas solicitantes $(1,045)$ menos de la mitad $(45.9 \%)$ obtuvieron recursos para sus proyectos.

Imagen 3. Empresas que han participado en el programa de estímulos scales, 2001-2006 (\%).

\begin{tabular}{|c|c|c|c|c|c|c|}
\hline \multirow[b]{2}{*}{ Año } & \multicolumn{5}{|c|}{ Empresas } & \multirow{2}{*}{$\begin{array}{c}\text { Estímulos obtenidos } \\
\text { Valor total } \\
\text { (miles de pesos) }\end{array}$} \\
\hline & Solicitantes & $\begin{array}{l}\text { Obtuvieron el } \\
\text { beneficio }\end{array}$ & $\begin{array}{l}\text { No obtuvieron } \\
\text { el beneficio }\end{array}$ & $\begin{array}{l}\text { Ya habían } \\
\text { participado }\end{array}$ & Nuevas & \\
\hline 2001 & 192 & 150 & 42 & 0 & 192 & 415.687 \\
\hline 2002 & 242 & 201 & 41 & 122 & 120 & 495.196 \\
\hline 2003 & 275 & 245 & 29 & 159 & 116 & 499.999 \\
\hline 2004 & 398 & 357 & 41 & 205 & 193 & 999.999 \\
\hline 2005 & 645 & 607 & 38 & 318 & 327 & 3.000 .000 \\
\hline 2006 & 1.045 & 480 & 565 & 305 & 175 & 4.000 .000 \\
\hline
\end{tabular}

Fuente: Dutrénit, G., M. Capdevielle, J.M. Corona Alcantar, M. Puchet Anyul, F. Santiago and A.O. Vera-Cruz. (2010, p. 194).

No obstante, a lo anterior, lo real es que el EFIDT representa un esquema de alto valor para los sectores productivos. En efecto es así, si se tiene en cuenta lo dicho por Cabrero cuando afirma que "Con el Estímulo Fiscal, aunado a otras políticas públicas, se espera incrementar este año la inversión privada en un 35.6 por ciento" (El Financiero, 2017, sección economía).
Imagen 4. Evolución de la composición del Gasto en Investigación Científica y Desarrollo Experimental (\%)

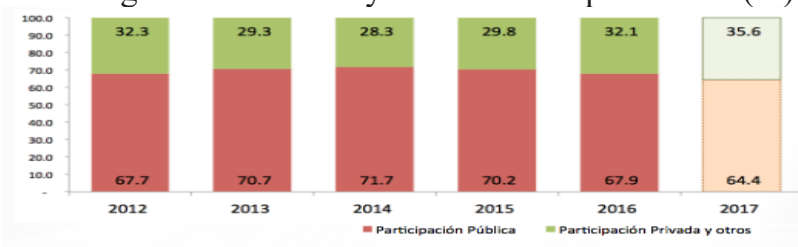
*Del 2015 al 2017 es estimación

Fuente: Sánchez (2017). 


\section{ii. Otros fondos CONACYT y sus alcances}

Otra práctica que cobra vigencia son los Fondos CONACYT (Fondos Sectoriales, Fondos Mixtos y Fondos Institucionales) que coadyuvan en la IDT en los sectores productivos y académicos y, que, sin lugar a duda, suman para cubrir con la demanda del sector privado que desean invertir en el desarrollo tecnológico de nuevos productos y procesos.

Imagen 5. Resumen general por tipo de fondo de la distribución de saldos bancarios de los fondos CONACYT

CIFRAS AL 31 DE DICIEMBRE ENTRE 20182020 (millones de pesos)

\begin{tabular}{|c|l|}
\hline Cantidad & Tipo de fondo \\
\hline 11 & INSTITUCIONALES \\
\hline 105 & MIXTOS \\
\hline $\mathbf{7 5}$ & SECTORIALES \\
\hline 3 & FIDETEC \\
\hline $\mathbf{1 9 4}$ & MONTO TOTAL $\quad \mathbf{\$ 7 3 . 2 9 9 , 4 2}$ \\
\hline
\end{tabular}

Fuente: Elaboración propia con información de la Dirección de Administración e Información de Fondos CONACYT con datos integrados por los Secretarios Administrativos de los Fondos CONACYT (Institucionales y Sectoriales) y la Unidad de Articulación Sectorial y Regional (Mixtos y Fordecyt), al cierre de diciembre 2020.

Estos fondos han representado entre 2018-2020 un total de $\$ 73.299,42$ millones de pesos, lo que permitió inyectar recursos a 194 proyectos en 4 fondos. Este monto se agrega a los ya múltiples recursos destinados a la $C \& T$, que bien puede ser significativo si se tiene en cuenta que la inversión privada tiene una participación baja en las actividades de IDT (en promedio 30\%), inclusive permitiría a que año con año los sectores productivos fijen rutas de trabajo y un esquema definido en el logro de sus propósitos.

Esta estrategia gubermamental es de suma importancia para los sectores productivos dado que es un incentivo de gran alcance que motiva a la inversión privada en materia de IDT a conjuntar esfuerzos con el sector público, no así para el gobierno en turno (2018-2024) pues hoy día esta política pública es un hecho pretérito con la extinción de 109 fideicomisos por la Cámara de Diputados el pasado mes de octubre de 2020, al tiempo, también, "se dispuso que la extinción de los fondos para C\&T se aplazará hasta junio de 2021" (El Financiero, 2020).

En el comunicado 297 del Senado de la República (2020), se recobran algunas intervenciones que se dieron en la discusión de extinción de los 109 fideicomisos "(...) que con esta reforma se permitirá una bolsa superior a los $68 \mathrm{mil}$ millones de pesos para atender los efectos de la pandemia (...) la ASF halló que los fideicomisos no tienen la obligación de ser transparentes, rendir cuentas y registrar egresos e ingresos en la cuenta pública, en el Presupuesto de Egresos ni en ningún otro registro público, además encontró discrecionalidad y abierta opacidad en su manejo, lineamientos y entrega de información sin estructura, entre otras observaciones (...) un estudio de Fundar, Centro de Análisis e Investigación, reveló que 93 por ciento de los recursos disponibles en fideicomisos, es decir, 905 mil millones de pesos no cuentan con controles de vigilancia (...) la extinción de 109 fideicomisos públicos representa un duro golpe a la ciencia, la tecnología, medio ambiente, cultura, salud, derechos humanos, entre otros (...) se trata de una reforma restrictiva, retroactiva y regresiva (...) es darle la espalda a víctimas, braseros, periodistas, artistas y científicos".

Esta extinción impuesta por la Cámara de Diputado es una política promovida por el Ejecutivo Federal basándose en su compromiso del combate a la corrupción que es eminentemente ideológico y no técnico según los partidos de oposición. Incluso se afirma que al no haber "un análisis profundo, con evidencias y sustentos sólidos por parte de las instituciones públicas, donde expongan la situación de cada uno de ellos para conocer sus debilidades y el impacto que tendrán en el mediano y largo plazo en la vida pública de México" (Senado de la República, 2020). Según el partido de Gobierno en voz del presidente de la Comisión de Hacienda y Crédito Público, que con esta extinción se logrará tener 
recursos económicos para la pandemia.

Al respecto, la directora general del CONACYT, señala “(...) tras el Decreto de extinción de fondos públicos, la Junta de Gobierno del Consejo formalizó el proceso de extinción de 61 fondos fiduciarios para ser concentrados y consolidados en uno solo, el Fordecyt-Pronaces, hacia el cual serán transferidos todos los recursos comprometidos en los fondos en proceso de desaparición. También explicó que los fondos sectoriales SENERConacyt (Hidrocarburos y Sustentabilidad) se encuentran en proceso de estudio y que el Fondo Cibiogem está excluido del Decreto (...) serán respetados todos los compromisos adquiridos por los fondos en proceso de extinción, garantizando desde el Fordecyt-Pronaces la ministración de recursos para los proyectos de investigación, infraestructura, becas o cualquier otra modalidad aprobada por los fondos (...) el respaldo irrestricto de la institución hacia los 26 Centros Públicos de Investigación del Conacyt para que conserven sus respectivos fondos, indispensables para su funcionamiento operativo y suministro de salarios, becas e insumos" (Dirección General CONACYT, Comunicado 159/20).

La extinción de algunos fondos afectan, entre otros, a múltiples Centros Públicos de Investigación (Colegio de la Frontera Norte (Colef), el Colegio de la Frontera Sur, el Cinvestav, el Instituto Mexicano de Tecnología del Agua (IMTA), el Centro de Investigación en Alimentación y Desarrollo (CIAD), el Centro de Investigación en Matemáticas, el Centro de Investigaciones Biológicas del Noroeste y el Centro de Investigaciones en Óptica), así como también la ministración "para el Fomento y Apoyo a la Investigación Científica y Tecnológica en Bioseguridad y Biotecnología -que auspicia el estudio de los riesgos de los organismos genéticamente modificados (transgénicos) en el medio ambiente- y de dos Fondos Sectoriales del Conacyt-Secretaría de Energía relacionados con la investigación, innovación y formación de recursos humanos de la industria petrolera" (Animal Político, 2 de octubre de 2020).

\section{iii. Programa Institucional 2018-2024 CONACYT (PI-CONACYT)}

Expuesto así, lo real es que la dinámica gubernamental emprendida por el gobierno en turno formuló el Programa Institucional 20182024 CONACYT (PI-CONACYT) teniendo como marco el Plan Nacional de Desarrollo (PND) 2019-2024 —como ha sido tradición en los gobiernos pasados - que a la letra señala “(...) el Conacyt reconoce la necesidad de generar políticas públicas con fundamento científico y con un enfoque articulado para el bienestar de la población, tal como lo señala el principio rector del PND 2019-2024. Bajo esta nueva perspectiva del Gobierno de México, el PND 2019-2024 establece como objetivo superior "El bienestar general de la población". Asimismo, define 12 Principios Rectores de alcance prioritario que orientarán al país hacia un desarrollo con bienestar, incluyente e igualitario" (PI-CONACYT, 2018).

En los prolegómenos del PI-CONACYT se percibe como una carga de pensamientos tal como aquellas líneas "El Conacyt tiene como prioridad orientar todas las acciones hacia el bienestar general de la población, sentando las bases que nos permitan alcanzar un verdadero cambio de régimen, modificando paradigmas e impulsando acciones articuladas en ese sentido y acordes a dichos principios rectores. No dejar a nadie atrás, no dejar a nadie afuera implica para el Conacyt poner a las humanidades, las ciencias, las tecnologías y la innovación al servicio del pueblo de México y de la atención de sus retos prioritarios"'s.

Posteriormente, se transita hacia un diagnóstico técnico —valioso para toda política pública (Bardach, 1999) — que transforma los referentes de antaño para introducir nuevas estrategias, acciones, metas e indicadores, incluso una estructura de cálculo de los parámetros. Este hecho es legítimo si se tiene en cuenta que los cursos de

3 El subrayado es de Mijael AltamiranoSantiago. 
acción del gobierno (cualquiera que sea su base ideológica o partidista) devienen de su propia visión, de sus propios referentes ideológicos y/o político, no así si se desea apuntalar políticas públicas de Estado en donde no cabe ni ideología, ni intereses particulares (o inicuos) y, menos aún, el término "cambio de régimen" que se anuncia en el epígrafe anterior.

Esto es así, si se tiene en cuenta lo escrito por Munárriz (2016) "Si la ciencia fuera una actividad tan pura y neutral como se pretende, los investigadores hubieran abandonado hace años muchas de las líneas de investigación y hubieran vuelto a los abandonados senderos del bosque a la búsqueda de paradigmas que fueran más consistentes con los datos que iban obteniendo. Pero podemos entender que múltiples presiones, básicamente corporativas y comerciales, han contaminado esta pureza ideal. No se trata de tirar a la basura todo lo que hemos aprendido estos años, sino de saber usarlo de una manera diferente. Encuadrando esos mismos datos, valiosísimos, en una teoría más útil. Que esté en crisis un paradigma no significa que tengamos disponible otro de repuesto. Habrá que abrir el foco antes de construir esta alternativa, que nacerá, por las mismas características del progreso de la ciencia, para ser sustituida por otra mejor. ¿Hacia dónde hemos de abrir ese foco?" (p. 295) ${ }^{4}$.

Nada más sólido y contundente para oponerse a lo anunciado en los prolegómenos de la PICONACYT en donde más que proponer un "cambio de régimen", lo que se debe hacer es transitar hacia una modificación o reestructuración 4 El subrayado es de Mijael AltamiranoSantiago. en forma y fondo en la gestación, elaboración e implementación de la política pública para, así, abandonar la visión de gobierno (o la visión ideológica/partidista) que ha dominado el proceso de toma de decisiones en los gobiernos de antaño y del presente (más aún ahora por la preeminencia discursiva del "combate a la corrupción" en los asuntos de gobierno) y, de ese modo, estacionarse en una política pública de Estado.

Esta afirmación viene a cuento por lo dicho de la directora general del CONACYT, quien mencional que la "ciencia occidental o neoliberal (...) se han confrontado con el pensamiento predominante acerca del quehacer cientifico"5 (infobae, 10 de octubre de 2020) o, en su caso, lo referido en el PI-CONACYT 2018-2024 en donde se denomina a los gobiernos anteriores como "gobierno neoliberal" o, en su caso, que "el neoliberalismo fue excluyente de oportunidades de formación de especialistas en ciencias, humanidades y tecnologías".

Sin entrar en esta discusión, lo que resulta necesario para el país es que la C\&T se le dé ese lugar que merece según la política comparada (Altamirano y Martínez, 2011) en donde se pone de manifiesto que este binomio ha ido más allá del 1\% del PIB recomendado por la UNESCO (ejemplo de ello, es Brasil que alcanza el 1.26\%; España el 1.24\%; Corea e Israel cerca del 5\%, Alemania y Estados Unidos en torno al 3\%) (OEI, 2020), inclusive da cuenta que éste ha conducido que los países sean competitivos en el concierto global (China, Corea del Sur, Japón, entre otros).

$5 \quad$ El subrayado es de Mijael AltamiranoSantiago. 
Imagen 6. Evolución porcentual en C\&T como proporción del PIB

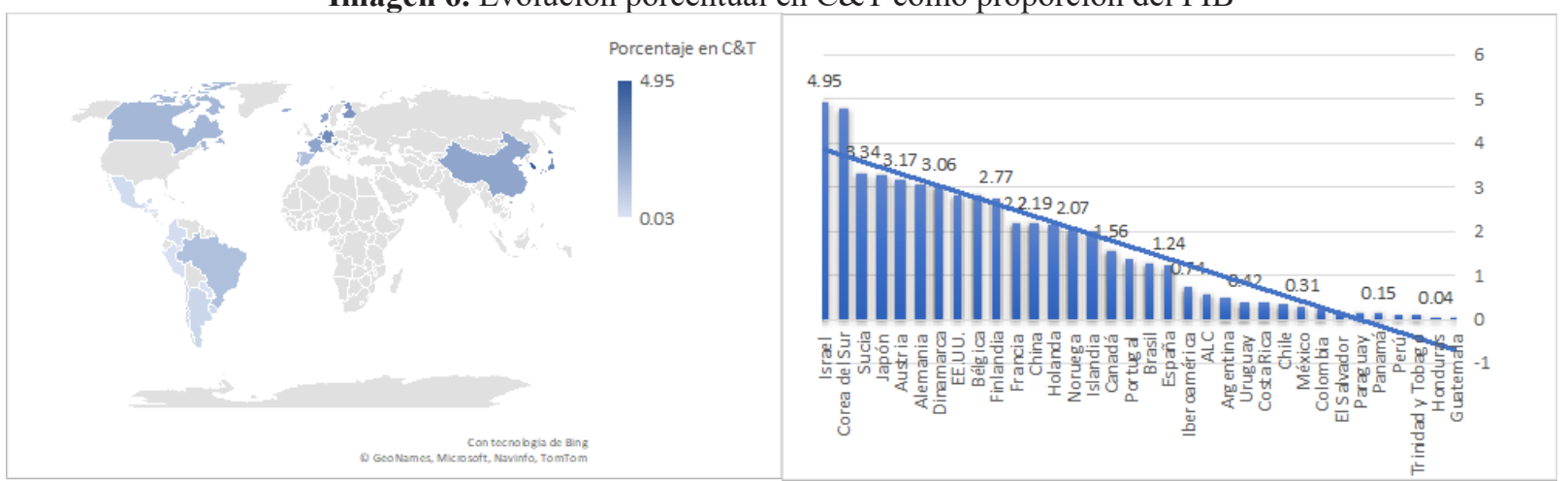

Fuente: OEI (2020, p. 23).

En adición al EFIDT y a los Fondos CONACYT, se suma el número de capital humano de alto nivel que ha formado el propio CONACYT a través de su Programa Nacional de Posgrado de Calidad (PNPC) que cuenta con 2,282 programas académicos en el 2018 y que hoy día (3/02/2021) se le ha agregado 153 más para sumar un total de 2,435 (Padrón del Programa Nacional de Posgrado de Calidad, 2021).

Imagen 7. Programas académicos PNPC por área del conocimiento del SNI

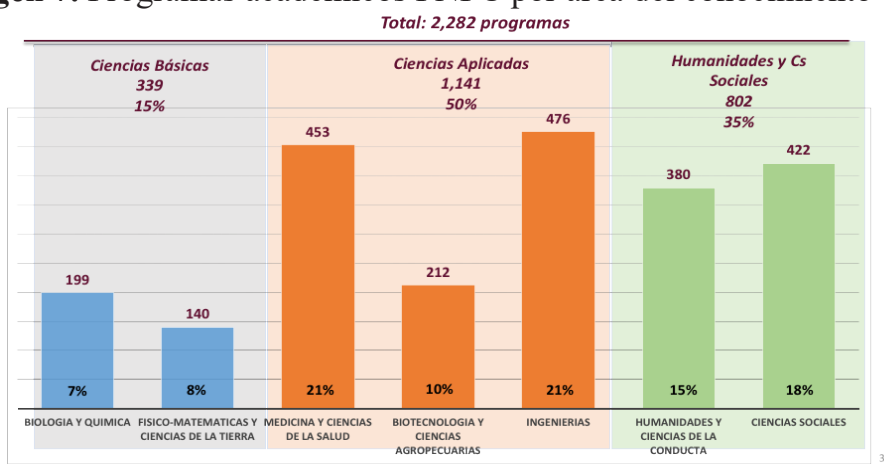

Fuente: Dirección de Posgrado, CONACYT, 2018.

Es menester señalar que, el PNPC es concomitante con el Programa de Becas Nacionales, que al mes de marzo de 2019 tuvo un total de 46,920 becarios y con una tasa de crecimiento porcentual anual de $2.2 \%$.

Imagen 8. Programas de Becas Nacionales

\begin{tabular}{|c|c|c|c|c|}
\hline \multicolumn{5}{|c|}{ Becas Nacionales } \\
\hline \multirow{2}{*}{ Modalidad de Becas } & \multicolumn{4}{|c|}{ Nivel de estudios } \\
\hline & Especialidad & Maestría & Doctorado & Total \\
\hline Becas de Posgrado Nacionales & 1.791 & 26.153 & 18.189 & 46.133 \\
\hline Becas Mixtas Nacionales & - & 1 & 8 & 9 \\
\hline Becas para Estancias Técnicas Nacionales & - & - & - & 34 \\
\hline Becas para Estancias Posdoctorales Nacionales & - & - & - & 667 \\
\hline Estancias Posdoctorales Nacionales Mujeres Indígenas & - & - & - & 6 \\
\hline Becas para Estancias Sabáticas Nacionales & - & - & - & 30 \\
\hline Becas para Retenciones y Repatriaciones & - & - & - & 41 \\
\hline \multicolumn{4}{|c|}{ Total Becas Nacionales } & 46.920 \\
\hline
\end{tabular}

Fuente: Informe de Actividades de CONACYT Enero-marzo 2019, p. 12.

TCP*: Tasa de Crecimiento Porcentual, respecto del mismo mes, durante el año pasado. 
Así, también, se agrega el Programa de Becas al Extranjero que tiene un total de becas asignadas de 4,337 y con una tasa de crecimiento porcentual anual de $1.3 \%$.

Imagen 9. Programas de Becas al Extranjero

\begin{tabular}{|c|c|c|c|c|}
\hline \multicolumn{5}{|c|}{ Becas Nacionales } \\
\hline \multirow{2}{*}{ Modalidad de Becas } & \multicolumn{4}{|c|}{ Nivel de estudios } \\
\hline & Especialidad & Maestría & Doctorado & Total \\
\hline Becas de Posgrado al Extranjero & 0 & 1.229 & 2.336 & 3.565 \\
\hline Becas Mixtas para estancias al Extranjero & 0 & 68 & 289 & 357 \\
\hline Becas para Estancias Técnicas al Extranjero & 0 & 0 & 0 & 30 \\
\hline Becas para Estancias Posdoctorales al Extranjero & 0 & 0 & 0 & 277 \\
\hline Becas para Estancias Sabáticas al Extranjero & 0 & 0 & 0 & 108 \\
\hline \multicolumn{4}{|c|}{ Total Becas Nacionales } & 4.337 \\
\hline
\end{tabular}

Fuente: Informe de Actividades de CONACYT Enero-marzo 2019, p. 12.

TCP*: Tasa de Crecimiento Porcentual, respecto del mismo mes, durante el año pasado.

Ambos programas, han estimulado la formación de nuevo capital humano con nivel doctorado sin que la iniciativa privada invierta en este rubro y, si, por el contrario, se vuelve una mano de obra calificada en la consolidación de los procesos de producción de éstas y de sus bienes de capital.

De ahí, que se demande que el sector privado sea un impulsor natural en materia de Gasto en Investigación y Desarrollo Experimental ${ }^{6}$ (GIDE) y, por consiguiente, se sume a la acción de gobierno en esta área para adecuarse al promedio de América Latina 0.55\% (GIDE/PIB), dado que históricamente el promedio ha estado en $0.51 \%$. Este porcentaje resultante del GIDE/PIB en México está muy alejado del promedio de la OCDE (2.35\%), la cual pertenece desde el 18 de mayo de 1994, siendo México el miembro número 25 en ese entonces.

Imagen 10. El GIDE en países seleccionados miembros de la OCDE (porcentaje)

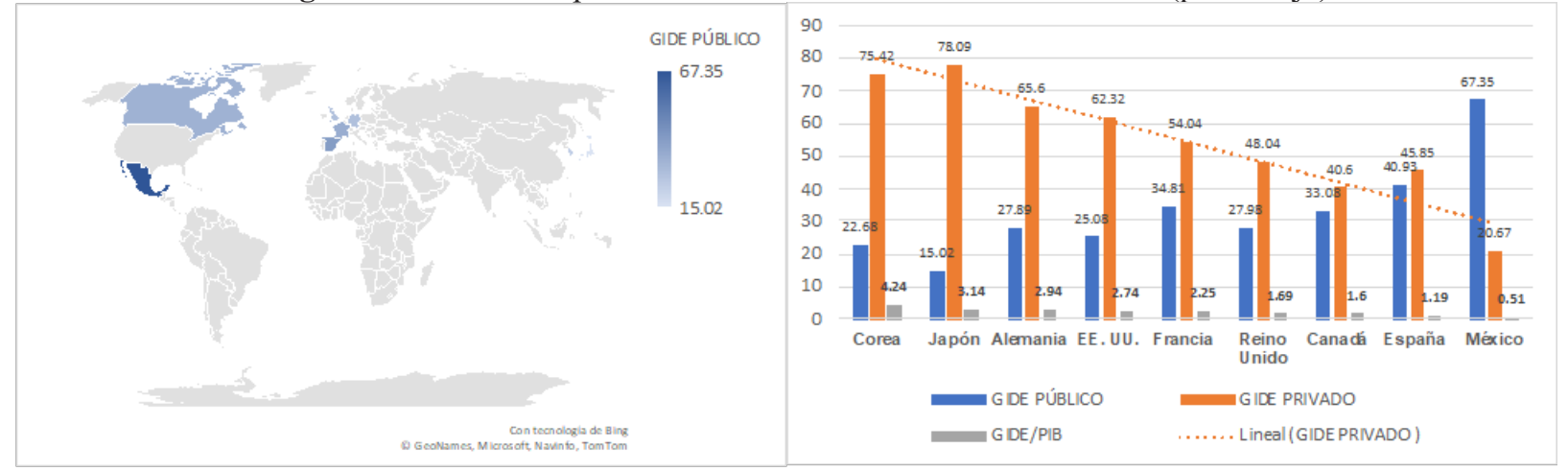

Fuente: CONACYT (2017, p. 21)

Este cúmulo de estrategias gubernamentales a efecto de que el sector productivo sea un actor de relevancia en el financiamiento del GIDE no ha dado frutos, pues la sinergia no se consolida y su avance es mínimo si se tiene presente los indicadores que maneja la OCDE (2016) al posicionar a México (20.67\%) en último lugar frente a sus similares de este Organismo, incluso por debajo

6 El GIDE es un componente del Gasto Nacional en Ciencia, Tecnología e Innovación que comprende exclusivamente aquellos recursos que se utilizan para generar nuevo conocimiento, excluyendo el gasto en otras actividades relacionadas como los servicios científicos y tecnológicos, las actividades de innovación; así como el gasto en educación y enseñanza científica y técnica, salvo el caso de los posgrados cuyo producto es un proyecto de investigación y desarrollo experimental (IDE) (CONACYT, 2017, p. 17). 
de Brasil (47.5\%), Colombia (46,59\%), Chile (35.84\%), Sudáfrica (38.90\%).

En lo referente a la C\&T hay que afirmar que subyace un desencuentro entre gobiernouniversidad, gobierno-empresa y universidadempresa al grado de ignorar que el desarrollo y la innovación tecnológica se da a partir de estas sinergias, incluso es sabido que la formación de profesionistas y cuadros para el desarrollo científico y tecnológico se funda de éstas (Arellano, 1996; Casas y Luna,1994). En la medida que el gobierno y las empresas se enlancen para hacer nodos en la universidad y, de ahí, se establezcan vínculos sólidos, el desarrollo y el progreso tecnológico no solamente estarán sustentados en la tecnología, sino también en el origen y construcción de las tecnologías creadas desde las universidades.

Al tiempo, también, el binomio empresauniversidad debe constituirse como una práctica recurrente y natural para que éste de igual forma pueda financiar y solventar las barreras estructurales, motivacionales y procedimentales que viven las universidades (Solleiro:1994, pp. 18-19) y, de ese modo, éstas sean el cimiento sobre el cual se edifiquen las políticas públicas en C\&T en el país, incluso robustecer el financiamiento que las empresas transfieren a la investigación universitaria, que a esta fecha no supera el 6 ó 7\% (Casas y Luna:1994).

Si bien es cierto que estos problemas son de antaño, actualmente se han acentuado, toda vez que el esfuerzo por acceder al proceso de globalización con ventajas competitivas, exige la instrumentación de un cúmulo de acciones consistentes e integrales para enfrentar los desafíos que impone la dinámica social, económica, científica, tecnológica o educacional.

Estas políticas públicas, además de las ya diseñadas, deben ser conducidas en varios frentes: i) establecer un sistema administrativo y reglas de operación simples para la asignación de los estímulos para invertir en C\&T (exenciones fiscales, bonos, entre otros); ii) incentivar a las universidades e institutos de investigación para que generen investigación con sentido social; iii) promover los sueldos y salarios competitivos a efecto de que el capital humano se aboque a la formación de investigadores; iv) equipar de instrumentos e infraestructura a los laboratorios de las universidades e institutos de investigación para no quedarse en la investigación básica; v) fortalecer los mecanismos formales (como el programa de repatración de capital humano de alto nivel) para vigorizar la infraestructura humana de las empresas y así, contribuir en la IDT; vi) fortalecer la cooperación internacional entre los agentes institucionales y los sectores productivos; vii) instrumentar mecanismos para enlazar el mercado con la investigación; viii) propiciar un nuevo formato cuádruple de vinculación (gobierno-empresa-universidadessociedad civil), etcétera.

La reestructuración del quehacer gubernamental debenecesariamente basarse en que la universidad, los centros y los institutos de investigación sean eje para el desarrollo de todo país y, en consecuencia, los investigadores/docentes representen una pieza fundamental para la transformación de las sociedades contemporáneas. Dejar de ver todo esto, implica de facto abandonar los postulados marcado por la teoría del crecimiento endógeno (Barro y Sala-i-Martin, 1995) y estar rezagado en el concierto de las naciones.

Por otro lado, el presupuesto federal ha hecho aún más complicado tener presente a la $C \& T$ en el espectro gubernamental, toda vez que los recursos asignados en los últimos sexenios (incluso este año de 2021) no alcanzan variaciones significativas como proporción del PIB.

La situación de la C\&T es aún grave, pues no es sólo en la asignación de recursos y en el número de programas y fondos institucionales vigentes, sino también en su gestión, si se tiene presente lo que alguna vez reportó Corona (2003, p. 59), al afirmar "(...) que algunos recursos han tenido baja utilización. Tal es el caso del crédito a cargo 
del Proyecto de Conocimiento e Innovación (PCI), en contrato con el Banco Mundial (núm. 4349-ME). El monto por 300 millones de dólares fue suscrito para atender una cobertura de 6 mil empresas, 10 mil estudiantes de posgrado y 5 mil investigadores, durante el periodo 1998-2003. Sin embargo, sólo se ha utilizado del orden de la mitad en el periodo 1998-2002 (1'556.6 millones de pesos)".

Esta dinámica que adquirió la gestión/ administración de los recursos por parte del CONACYT en esos cuatro años, además de que imposibilitó el cumplimiento de una serie de tareas contraídas con el organismo mundial - continúa Corona (2003, p. 59)— obligó al gobierno federal a pagar 13 millones de dólares en penalizaciones por subejercicio del crédito contratado por el CONACYT con el Banco Interamericano de Reconstrucción y Fomento (multas a CONACYT por subejercicio de un préstamo, JOR-1522002).

Las implicaciones de este subejercicio y todo lo que se deriva de la financiación de la C\&T, obligan a replantear la política pública sobre esta materia para fortalecer los mecanismos, elementos e insumos que subyacen en su labor para, de ese modo, hacer de este tema una actividad dinámica, creciente y con altos rendimientos para los sectores involucrados y, particularmente, para el propio país.

Hoy día, la inercia adquirida en materia de C\&T en el país, además de no tener la preeminencia en los asuntos de gobierno (verbi gratia, el órgano rector desde su creación (CONACYT) -27 de diciembre de 1970 - no ha dejado de ser una dirección general, la cual le imposibilita tener la dimensión de una secretaría de estado, incluso hace que se configuere como apéndice, sea de la oficina de la presidencia de la República o de la propia Secretaría de Educación Pública -SEP- y en el proceso de toma de decisiones, en su conducción y su quehacer se ha privilegiado el elemento ideológico-político y no a las necesidades reales que exige el país. De ahí que este binomio no haya tenido desde entonces una dinámica propia, que si tienen en otros países, sin sujetarse al vaivén de los gobiernos en turno. Los avances logrados son insuficientes pues no se han apuntalado políticas estructurales y sólidas que incentive a los diferentes sectores empresarialesproductivos y no-lucrativos, entre otros, para impulsar una estrategia integral en esta área.

\section{CONCLUSIONES}

La posición de México en materia de C\&T no es nada alentador, no sólo por su desdén ante la recomendación hecha por la UNESCO y a su nulo interés por reconocer a este binomio como base de transformación de las economías postindustriales, sino también al rol que éste adquiere en los asuntos públicos.

La C\&T está lejos de ser un asunto que se vea desde una perspectiva de Estado dado que el trato recibido por los gobiernos de antaño y el presente ha dominado una visión ideológica, que más que ayudar, ha llevado a este binomio a rezagarse $\mathrm{y}$, en consecuencia, no sea visto como promotor de una dinámica económica creciente y competitiva (caso Corea del Sur).

Este abandono por configurar una política pública de Estado en C\&T hace que México no figure frente a sus pares de la OCDE y de sus socios comerciales en los grandes agregados en C\&T (actividad científica, tecnológica e innovación; acervo de recursos humanos en C\&T; tasa de dependencia; publicación ISI; gasto interno bruto en I+D; activos intangibles; entre otros). En tanto no se conciba a la C\&T desde la óptica del gobierno como eje rector de desarrollo y crecimiento económico, difícilmente habrá un viraje en la realidad nacional, y sólo imperará el discurso en el proceso de toma de decisiones, sin materializarse en pro del país. Al tiempo que, imposibilitará que México se acerque al nivel de sus similares miembros de la OCDE.

Los indicadores de la OCDE en materia de desarrollo tecnológico y conocimiento están 
distantes de los estándares de México. No tenerlos presente en el diseño de las políticas públicas, la brecha será aún más grande. Hoy por hoy se deben buscar directrices que impulsen nuevos escenarios para la C\&T no sólo en lo legal, sino en sus componentes, agregados, programas, acciones e instancias.

\section{REFERENCIAS}

1. Altamirano, M. y Martínez, A. (2011). El método comparado y el neo-institucionalismo como marco metodológico para la investigación en las Ciencias Sociales. Revista Mundo Siglo XXI. VII (25), 55-63. México: CIECAS-IPN.

2. Altamirano, M. (2010). Ciencia y tecnología: México desde una perspectiva comparada. Mario Sánchez Silva (coord.) Economía del conocimiento y globalización. México: CIECAS-IPN.

3. Animal Político (2 de cotubre de 2020). Recuperado 2 de febrero de 2021, de https://www.animalpolitico.com/2020/10/titular-conacyt-defiende-desaparicion-fideicomisos/.

4. Bardach, E. (1999). Los ocho pasos para la política pública, CIDE-Porrúa.

5. Barro, R. J. y Sala-i-Martin, X. (1995). Economic Growth, USA, McGraw-Hill.

6. Blanchard, O. y J. Fischer (1989). Lectures on Macroeconomics, Cambridge, MIT Press.

7. Cámara de Diputados. (27/9/2011). Que reforma el artículo 219 de la Ley del Impuesto Sobre la Renta, a cargo del diputado Alberto Emiliano Cinta Martínez del grupo parlamentario del PVEM, y suscrita por integrantes de la comisión especial para la competitividad. http://sil.gobernacion. gob.mx/Archivos/Documentos/2011/09/asun_2798283_20110927_1317142864.pdf.

8. Cárdenas, M., Parra, L., Picón, J., Pineda, H., y Rojas, R. (2007). Las Representaciones Sociales de la Política y la Democracia. Última Década. 15(26), 53-78. ttp://dx.doi.org/10.4067/S071822362007000100004

9. Casas, R. y Luna, M (1994). Condicionantes políticos de la nueva relación entre la universidad e industria, en Campos, M. y Corona, L. Universidad y vinculación: nuevos retos y viejos problemas, IIMAS-UNAM, México.

10. CONACYT (2017). Informe General del Estado de la Ciencia, la Tecnología y la Innovación. México: CONACYT. Recuperado 4 de febrero de 2021, de https://www.siicyt.gob.mx/index.php/ transparencia/informes-conacyt/informe-general-del-estado-de-la-ciencia-Tecnología-e-innovacion/informe-general-2017/4813-informe-general-2017/file.

11. CONACYT (9/11/2020). Recuperado 9 de noviembre de 2020, de https://www.conacyt.gob.mx/ index.php/comunicados/1311-aviso-aclaratorio-bioTecnología-si-esta-incluida-en-sni.

12. CONACYT. (2020a). Estímulo Fiscal a la Investigación y Desarrollo de Tecnología (EFIDT). Recuperado 27 de enero de 2021, de https://www.conacyt.gob.mx/index.php/fondos-y-apoyos/ estimulo-fiscal-a-la-investigacion-y-desarrollo-de-Tecnología-efidt.

13. Corona, L. (2003). El nuevo CONACYT ante las metas del programa especial de Ciencia Tecnología, Eseconomía, n. 5, México, Escuela Superior de Economía-IPN, pp. 55-61.

14. Dirección General CONACYT, Comunicado 159/20. Los fideicomisos del Conacyt serán consolidados en un solo fondo, el Fordecyt-Pronaces. Recuperado 27 de enero de 2021, de https://www. conacyt.gob.mx/index.php/comunicados/1277-com159-2020.

15. Dirección de Posgrado, CONACYT, (2018).

16. Dutrénit, G., M. Capdevielle, J.M. Corona Alcantar, M. Puchet Anyul, F. Santiago and A.O. VeraCruz. (2010). El sistema nacional de innovación mexicano: estructuras, políticas, desempeño y desafíos. México: UAM/Textual S.A. Recuperado 27 de enero de 2021, de https://mpra.ub.unimuenchen.de/31982/1/MPRA_paper_31982.pdf.

17. El Financiero (08/10/2020). ¿Tienes una empresa? Accede al estímulo fiscal por investigación. Sección Economía. Recuperado 27 de enero de 2021, de https://www.elfinanciero.com.mx/nacional/camara-de-diputados-aprueba-dictamen-que-elimina-fideicomisos-pasa-al-senado.

18. El Financiero (09/05/2017). ¿Tienes una empresa? Accede al estímulo fiscal por investigación. Sección Economía. Recuperado 27 de enero de 2021, de https://www.elfinanciero.com.mx/econo$\mathrm{mia} /$ tienes-una-empresa-accede-al-estimulo-fiscal-por-investigacion.

19. Infobae (10 de octubre de 2020). Recuperado 2 de febrero de 2021, de https://www.infobae.com/ america/mexico/2020/10/10/califica-a-la-ciencia-como-neoliberal-y-la-llegada-a-la-luna-comoinutil-las-polemicas-frases-de-la-directora-de-conacyt/. 
20. Informe de Actividades de CONACYT (2019). Enero-marzo.

21. LJA.MX. (26/11/2019). Se reduce $0.5 \%$ el presupuesto al Ramo 38, para CONACYT. Recuperado 28 de enero de 2021, de https://www.lja.mx/2019/11/se-reduce-0-5-el-presupuesto-al-ramo38-para-conacyt/.

22. Mariluz, R. (25/09/2020). Niega Conacyt que haya borrado Biotecnología de nuevo reglamento. Sección Nación. Recuperado 27 de enero de 2021, de https://asillarota.com/nacion/niega-conacyt-que-haya-borrado-bioTecnología-de-nuevo-reglamento/438101.

23. Martuscelli, J. (2012). Programa de Estímulos Fiscales: un diagnóstico. Restricciones e incentivos a la innovación en México. México: Centro de Estudios Sociales y de Opinión Pública-Cámara de Dipuados.

24. MORENA (2014). Declaración de Principios. Recuperado 27 de enero de 2021, de https://morena. si/wp-content/uploads/2014/12/declaracion-de-principios-de-morena1.pdf

25. Moscovici, S. (1979). El psicoanálisis, su imagen y su público, Buenos Aires: Huemul.

26. Munárriz, M. (2016). Cambio de régimen, cambio de paradigma. Revista de la Asociación Española de Neuropsiquiatría, 36(130), 293-296. Recuperado 03 de febrero de 2021, de http://scielo. isciii.es/scielo.php?script=sci_arttext\&pid=S0211-57352016000200001\&lng=es\&tlng=es.

27. OEI. (2020). EL ESTADO DE LA CIENCIA. Principales Indicadores de Ciencia y Tecnología Iberoamericanos. Recuperado 2 de febrero de 2021, de http://www.ricyt.org/wp-content/ uploads/2020/11/ElEstadoDeLaCiencia_2020.pdf.

28. Padrón del Programa Nacional de Posgrado de Calidad. (2021). Recuperado 3 de febrero de 2021, de http://svrtmp.main.conacyt.mx/ConsultasPNPC/padron-pnpc.php.

29. PI-CONACYT. (2018). Programa Institucional 2018-2024 del Consejo Nacional de Ciencia y Tecnología. Diario Oficial de la Federación. Recuperado 27 de enero de 2021, de https://www.dof. gob.mx/nota_detalle.php?codigo $=5595309 \&$ fecha $=23 / 06 / 2020$.

30. Piña, J. M. y Cuevas, Y. (2004). La teoría de las representaciones sociales: Su uso en la investigación educativa en México. Perfiles educativos. vol.26, n.105-106. Recuperado 28 de enero de 2021, de http://www.scielo.org.mx/scielo.php?script=sci_arttext\&pid=S0185-26982004000100005\&ln $\mathrm{g}=\mathrm{es} \& \mathrm{nrm}=$ iso.

31. Porter, Michael E. (1990). The competitive advantage of nations, Harvard Business Review, vol. 68, no. 2, marzo-abril, pp. 73-93.

32. Powell, W. \& Snellman, K. (2004). The Knowledge Economy. Annual Review of Sociology, 30 (1).

33. Ricardo, D. (1993). Principios de economía política y tributación. Bogotá: Fondo de Cultura Económica.

34. Ritzer, G. (2007). The Coming of Post-Industrial Society. New York: McGraw-Hill.

35. Romer, D. (1996). Advanced Macroeconomics, Nueva York, McGraw-Hill.

36. Ruelas, I. (13/12/2019). Gasto público en ciencia y tecnología en México, ¿por qué, ¿cómo y para qué? Nexos. Recuperado 27 de enero de 2021, de https://educacion.nexos.com.mx/?p=2073.

37. SaKong, I. y Koh, Y. (Ed.). (2010). La economía coreana Seis décadas de crecimiento y desarrollo. Santiago de Chile: CEPAL, KDI, KCLAC.

38. Sánchez, V. (8/5/2017). Lo que debes saber de los estímulos fiscales. CienciaMX. Recuperado 27 de enero de 2021, de http://www.cienciamx.com/index.php/sociedad/politica-cientifica/15109-loque-debes-saber-de-los-estimulos-fiscales.

39. Sartori, G. (2005). ¿Qué es la democracia? México: Taurus-Pensamiento.

40. Senado de la República. (21/10/2020). Aprueba Senado, en lo general, extinción de 109 fideicomisos. Boletín. número 297. Recuperado 2 de febrero de 2021, de http://comunicacion.senado.gob. $\mathrm{mx} /$ index.php/informacion/boletines/49479-aprueba-senado-en-lo-general-extincion-de-109-fideicomisos.html.

41. SHCP. (2021). Proyecto de Presupuesto de Egresos de la Federación 2021 Estrategia Programática. Recuperado 27 de enero de 2021, de https:/www.ppef.hacienda.gob.mx/work/models/PPEF2021/ docs $/ 38 /$ r38_ep.pdf.

42. Smith, A. (2018). Investigación sobre la naturaleza y causas de la riqueza de las Naciones. México: Fondo de Cultura Económica.

43. Smith, A. (2019). La Riqueza de las Naciones (Libros I-II-II y selección de los libros IV y V). Madrid: Editorial Biblioteca Nueva. Madrid.

44. SNI-CONACYT. (2019). Áreas del SNI. México: CONACYT. 
45. Solow, R. M. (1956). A Contribution to the Theory of Economic Growth. The Quarterly Journal of Economics. 70(1).pp. 65-94. Recuperado 27 de enero de 2021, de https://doi.org/10.2307/1884513 46. Toffler, A. (1980). The Third Wave. USA: Bantam. 